\title{
Prediction Of Lung Cancer Using Image Processing Techniques: A ReView
}

\author{
Arvind Kumar Tiwari \\ GGS College of Modern Technology, SAS Nagar, Punjab, India
}

\begin{abstract}
Prediction of lung cancer is most challenging problem due to structure of cancer cell, where most of the cells are overlapped each other. The image processing techniques are mostly used for prediction of lung cancer and also for early detection and treatment to prevent the lung cancer. To predict the lung cancer various features are extracted from the images therefore, pattern recognition based approaches are useful to predict the lung cancer. Here, a comprehensive review for the prediction of lung cancer by previous researcher using image processing techniques is presented. The summary for the prediction of lung cancer by previous researcher using image processing techniques is also presented.
\end{abstract}

\section{KEYWORDS}

Classification, lung cancer, accuracy, image processing techniques

\section{INTRODUCTION}

Lung Cancer is the uncontrolled growth of abnormal cells, start off in one or both lungs, usually in the line the air passages. The abnormal cells do not develop into healthy lung tissue; they provide rapidly and form tumours. According to American cancer society the cases of lung cancer increases very rapidly and almost $14 \%$ newly diagnosed cancers are a lung cancer and also the main cause of cancer death worldwide. The previous study of diagnosis showed that the most of the lung cancer patients belongs to the age of 60 years.

Lung Cancer is one of the most serious human body problems in the world. The death rate of lung cancer is highest of all other types of cancer. The survival rate of lung cancer is very smallest among all types of cancer. So, there is a need to design a computational intelligence based approaches to detect the lung cancer because the survival from lung cancer is directly related to its growth at its detection time. If we detect lung cancer at early stage, then there are more possibilities to survive the patients. It is also showed from previous study that cigarettes smoking are the main cause of lung cancer. It is observed that an estimated $85 \%$ of lung cancer cases in males and $75 \%$ lung cancer cases in females where cigarette smoking is the main reason

\section{LiterATURE SURVEY}

In literature various researchers have been used image processing techniques to predict the lung cancer. Sharma et.al. (2011) used lung CT images extracted from NIH/NCI Lung Database Consortium and proposed an automatic computer aided diagnosing system for detection of lung cancer by analyzing these lung CT images. The authors of the paper have used several steps for the detection of lung cancer. Firstly, they extracted the lung region from the computer tomography image using various image processing techniques such as bit image slicing, erosion and wiener filter. In the first step the bit image slicing technique was used to convert the CT 
images into a binary image then after extraction the region growing segmentation algorithm was used for segmenting the extracted lung regions. After segmentation of lung region they used rule based model to classify the cancer nodules. Finally, a set of diagnosis rules were generated from the extracted features and with the help of diagnostics indicator. It was observed that the proposed method achieved the overall accuracy of 80\%. Anam Tariq et.al. (2013) has developed a computerized system, that was detected the lung nodules with the help of CT scan images. The computerized system consists of two stages, first one is lung segmentation and enhancement and second one is feature extraction and classification. For removing background and extracts the nodules from an image, the threshold segmentation technique was applied. When extraction and segmentation were completed, then a feature vector was used to -calculate the abnormal region. After that the regions were classified using neuro fuzzy classifier. This system gives the facility to detect the smallest nodules which lead to early diagnosis of lung cancer.

Sundararajan et.al. (2010) have been proposed support vector machine for the detection of pneumoconiosis by using various textural features for disjoint segments of the lung and focused on a subset of lung disorders. Le (2011) has been proposed a complex system for the detection of various lung disorders and image processing techniques were used for the detection of lung nodule but they were not used false positive reduction step. Chaudhary et.al. (2012) aim has to get more accurate results using the various enhancement and segmentation techniques. The image processing procedures that is, image pre-processing; segmentation and feature extraction were done. In image enhancement, image are compared with Gabor filter, auto enhancement and fast Fourier transform techniques. In the segmentation stage the Watershed and thresholding segmentation were used and comparison has been made. Hashemi et.al. (2013) his aim has to improve the efficiency of the lung cancer diagnosis system, with the help of proposing a region growing segmentation method to segment CT scan lung images. First of all, for noise removing linear-filtering and contrast enhancement technique was used as pre-processing step, to prepare the image for segmentation. After that for differentiating among malignant, benign and advance lung nodules, the cancer recognition was presented by fuzzy inference system. The authors also compare the diagnosis performance of the proposed method with the artificial neural network.

Schilham et.al. (2006) have been proposed to use a k-nearest neighbor (k-NN) classifier by using the images from the JSRT database for searching the Gaussian scale space maxima in multi-scale to detect different sized lung nodules. It was also observed that the proposed method performed better only at higher false positive rates. Pereira et.al. (2007a) has been proposed a sliding band filter based on the convergence of radial gradients to detect the lung nodule by using the JSRT database without a false positive reduction step. S.K. Vijai Anand et.al. (2010) have proposed a system which was predicted lung tumor from CT images with the help of image processing techniques coupled with neural network classification, to recognize whether benign or malignant. Then optimal thresholding was applied for noise removal to segregate lung regions. To segment the lung nodules region growing method was used. After that, back propagation neural network was used to classify as cancerous or non-cancerous. S.L.A. Lee et.al. (2008) have been proposed a random forest based classifier to detect all the nodules in the images and recorded a low false detection rate. The proposed method includes three stages such as image acquisition, background removal and nodule detection for identification of lung nodules by using images from the LIDC lung databases. The performance of the system was observed on some images containing nodules and some images containing no-nodules that were normally selected from database images.

Vijay A. Gajdhane et.al. (2014) have been proposed detection of lung cancer by using various image processing techniques with the help of CT scan images. There were mainly three processes used throughout the report; pre-processing step, feature extraction step, and finally the classification step. In pre-processing step noise was removed with the help of median filter, and Gabor filter was used for image enhancement and segmentation. Area, perimeter, and eccentricity were the extracted features. The extracted ROI (Region of Interest) transformation function was 
created an array of ROI. At last, Support Vector Machine (SVM) was used for classification purpose. Fan Zhang et.al. (2013) have been proposed Support Vector Machine (SVM) based classifier by using feature based imaging classification method to classify the lung nodules in Low Dose Computed Tomography (LDCT) slides into four categories that is, well circumscribed, vascularized, juxta-pleural and pleural-tail. The proposed support vector machine based method was trained with the help of polynomial kernel by C-SVC and the probability estimates upon the different types were predicted with the obtained SVM model which was used to classify the feature descriptors into four categories. Lo et.al. (1995) applied a matched filter and sphere template matching to enhance round-shaped objects, and filtered false results with a convolutional neural network (CNN). They measured good performance on a small set of images. S. Sivakumar et.al. (2013) have proposed an efficient lung nodules detection scheme by performing nodule segmentation. For nodule segmentation weighted fuzzy probabilistic based clustering was carried out for lung cancer images. SVM was. used for classification purpose. Iiya Levner et.al.(2007) have proposed the novel approach that was created the topographical function and object markers used within watershed segmentation. The watershed algorithm was commonly used within the unsupervised setting of segmenting the image into a set of non-overlapping regions. Yoshida et.al. (1995) have been proposed a combination of two enhancers such as a difference image algorithm and wavelet transform sensitive to subtle nodules. The proposed method integrated overlapping and lesion models were used in combination. It was observed that the proposed model obtained better result in compared to single algorithm without any incorporation of false positive reduction technique. Sunil Kumar et.al.(2014) have proposed Computer aided diagnosis (CAD) that was detected the lung cancer at early stage by using various image processing techniques with the help of CT scan images. In pre-processing step noise was removed and converted the image into gray scale. Histogram thresholding was used for thresholding purpose. Finally, region growing algorithm was used for classification purpose. Xu et.al. (1997) proposed another difference image algorithm. For false positive reduction, they created a hybrid system with the help of an artificial neural network (ANN) and a rule-based technique. M. Arfan Jaffar et.al. (2008) have presented a method for lung segmentation based on Genetic Algorithm (GA) and morphological image processing techniques. GA was applied on the normalized histogram to determine the threshold to separate out background and object. When background was removed the morphological operation was performed in three operations i.e. to filter noise, to smooth the image and to detect edges.

Giger et.al. (1990) have been proposed a difference image technique by using image processing techniques to create a nodule enhanced and a nodule suppressed image. They found that the proposed method eliminates everything remaining the shadow of nodule-like objects. They used less powerful methods for classification by using various geometry features to leave out false findings. Hiram Madero Orczco et.al. (2013) have been proposed a SVM based method by using eight texture feature that wre extracted from histogram and the gray level concurrence matrix to classify the image with nodules or without nodules without considering the segmentation stage. Fatma Taher et.al. (2012) have been proposed a combination of Bayesian classification and a Hopfield Neural Network (HNN) for extracted and segmented the sputum cells for a diagnosis of lung cancer. It was observed that The HNN segmentation algorithm outperforms the Fuzzy CMean clustering, that allow the extraction of nuclei and cytoplasm regions successfully. For improved the performance of HNN algorithm, morphological was processing on the segmented image. Kesav Kancherla et.al. (2013) have proposed an early lung cancer detection methodology with the help of Nucleus Based features. To perform nucleus segmentation the seeded region growing segmentation technique was used. An additional criterion like nucleus size to seeded region growing method was used for better accuracy. JIA Tong el.al. (2007) have been presented an automatic computer-aided detection(CAD) scheme, that could identify the pulinonary nodule at an every stage from CT images. An important pre-processing step of lung cancer was an improved optimal gray-level Threshold segment lung parenchyma. Region growing algorithm was proposed for Trachea and Main Airway Bronchi Elimination then the filter of nodule 
candidate, the detection of nodule candidate, the feature extraction and classification and threedimensional visualization was done. The accuracy of the CAD system was $95 \%$ which indicate good performance.

Penedo et.al. (1998) have been proposed a neural network based method for nodule enhancement by using another ANN to select only true findings and the results were presented on a moderatesized, private database extended by simulated nodules. Mao et.al. (1998) has evaluated the nodule enhancing capabilities of fragmentary window filtering. The authors were presented preliminary results on a few generated radiographs M. Gomathi \& P. Thangaraj et.al. (2010) have been proposed a support vector machine based (CAD) system for automatically detection of the lung cancer nodules with reduction in false positive rates. They used various image processing techniques to obtain the lung regions from $\mathrm{CT}$ scan images such as the segmentation was carried to apply fuzzy possibility C Mean (FPCM) clustering algorithm. Amjed S. Eslam b Jaber et.al. (2014) have been proposed an automatic computer aided diagnosis (CAD) system that includes three steps such as thresholding the CT image by segmentation, labeling the founded regions and regions are extracted for further analysis for the detection of lung cancer using CT scan images. Ghayoumi H, Siamak J et.al. (2013) have been proposed an image analysis approach for automated detection, segmentation and classification of abnormal cancer cells from normal cells was introduced. Gaussian smoothing was used for detection and segmentation. At last Fast Fourier Transforms (FFT) was used for extracted the position of the objected cells. Patil, Kuchanur et.al. (2012) have been proposed a system to identify the lung nodules by extracted the various geometrical and textural features like area, perimeter, entropy etc. using various image processing techniques. When geometrical and textural features were extracted then these features were applied for the classification of lung cancer.

Muhm et. al. (1983), Quekel et. al. (1999), Shah et. al. (2003) and Doi (2007) mentioned that many of the tumors were overlooked by radiologists in the early stage. Prashant Naresh et.al .(2014) have predicted lung cancer at initial stage by using various image processing and data mining techniques. He was proposed an automated diagnosis system for the prediction of lung cancer. Image acquisition, noise removal, segmentation, and morphological operations were used in image processing for information retrieval. Kobayashi et.al. (1996) MacMahon et.al. (1999) and De Boo et.al. (2009) studied that the efficiency of lung screening can be improved by using a computer aided diagnosis system, while Hoop et.al. (2010) warns that further training of the users may be important, as they often find it hard to differentiate between true and false findings of the machine. Ada (2013) has been proposed a method that is based on the combination of feature extraction and Principal Component Analysis (PCA) for lung detection with the help of CT scan images by using the histogram equalization for per-processing step of the images and features was extracted with the help of PCA. Sudha. V, Jayashree. P et.al. (2012) have been also proposed an efficient lung nodule detection system by using CT scan images. The proposed method performed two steps; first of all lung region segmentation through thresholding and then thresholding and morphological operations were used segmenting the lug nodules. Weng et.al. (2009) have been proposed a method based on a difference image technique and SVM classification by using the statistics of gradients as features but they also used JSRT image database for testing. So, they obtained the performance of the proposed method on the merged database. Snoeren et.al. (2010) have been proposed a neural network based on gradient convergence based method and location features to train a proposed model with simulated nodules without using a false positive reduction step. 
Advanced Computational Intelligence: An International Journal (ACII), Vol.3, No.1, January 2016

\section{Summary For The Classification Of Lung Nodule}

In Literature, it is observed that the image processing techniques with the computational intelligence based approaches are useful for the prediction and decision making of lung cancer. Table 1 gives the summary of image processing techniques and classification approaches with their performance analysis for the detection of lung nodules.. The summary of lung nodule detection and classification is presented in Table-1.

Table-1 Summary for the classification of lung nodule

\begin{tabular}{|c|c|c|c|}
\hline AUTHOR & IMAGES & $\begin{array}{l}\text { CLASSIFICATION } \\
\text { TECHNIQUE }\end{array}$ & ACCURACY \\
\hline Disha Sharma & $\mathrm{CT}$ & Diagnostic Indicators & $80 \%$ \\
\hline Anam Tariq & $\mathrm{CT}$ & Neuro-Fuzzy & $95 \%$ \\
\hline $\begin{array}{l}\text { F.Paulin } \\
\text { Dr.A.Santhakumaran }\end{array}$ & $\mathrm{CT}$ & $\begin{array}{l}\text { Back propagation } \\
\text { algorithm used for } \\
\text { training Multilayer } \\
\text { Perceptron(MLP) }\end{array}$ & $99.28 \%$ \\
\hline Yang Liu & CT & SVM(GRBF kernel type) & $87.82 \%$ \\
\hline $\begin{array}{l}\text { Yao ying huang,wang } \\
\text { sen li ,Xiaojiao ye }\end{array}$ & CT & $\begin{array}{l}\text { Genetic algorithm, feature } \\
\text { selection }\end{array}$ & $99.1 \%$ \\
\hline Dr.K .Usha rani & $\mathrm{CT}$ & $\begin{array}{l}\text { Feed forward, Back } \\
\text { propagation }\end{array}$ & $92 \%$ \\
\hline Afzan Adam & $\mathrm{CT}$ & $\begin{array}{l}\text { Genetic algorithm and } \\
\text { Back propagation neural } \\
\text { network }\end{array}$ & $83.36 \%$ \\
\hline S.K Vijai Anand & CT & $\begin{array}{l}\text { Back propagation network } \\
\text { classification }\end{array}$ & $86.30 \%$ \\
\hline David B.fogel & CT & $\begin{array}{l}\text { Back propagation network } \\
\text { classification }\end{array}$ & $98.2 \%$ \\
\hline JR Marsilin & $\mathrm{CT}$ & SVM & $78.00 \%$ \\
\hline Li Rong,Sunyuan & CT & SVM-KNN classifier & $98.06 \%$ \\
\hline F Eddaoudi & CT & SVM & $95 \%$ \\
\hline Aparna Kanakatte & PET & k-NN, SVM & $97 \%$ \\
\hline $\begin{array}{l}\text { S. Aruna, } \\
\text { Dr S.P. Rajagopalan }\end{array}$ & CT & SVM & $98.24 \%$ \\
\hline S.Sivakumar & CT & SVM(RBF kernel type) & $80.36 \%$ \\
\hline $\begin{array}{ll}\text { Hiram } & \text { Madero } \\
\text { Orozco } & \end{array}$ & $\mathrm{CT}$ & SVM & $84 \%$ \\
\hline Fatma Taher & Sputum & Bayesian & $88.62 \%$ \\
\hline Kesav Kancherla & Sputum & Random forest(bagging) & $87 \%$ \\
\hline Tuba kiyan & $\mathrm{CT}$ & Radial basis function & $96.81 \%$ \\
\hline
\end{tabular}


Advanced Computational Intelligence: An International Journal (ACII), Vol.3, No.1, January 2016

\section{OBSERVATION AND RECOMMENDATION}

$>$ Using CT images the SVM classification technique achieved accuracies between $78 \%$ to $98.24 \%$.

$>$ Using CT images the Back Propagation Network classification technique achieved accuracies between $86.30 \%$ to $99.28 \%$.

$>$ Using CT images the Neuro Fuzzy classification technique achieved accuracy $95 \%$

$>$ Using Sputum the Bayesian classification technique achieved accuracy $88.62 \%$.

$>$ Using CT images the Genetic Algorithm classification technique achieved accuracies between $83.36 \%$ to $99.10 \%$.

\section{Conclusion}

Prediction of lung cancer is most challenging problem due to structure of cancer cell, where most of the cells are overlapped each other. The image processing techniques are mostly used for prediction of lung cancer and also for early detection and treatment to prevent the lung cancer. To predict the lung cancer various features are extracted from the images therefore, pattern recognition based approaches are useful to predict the lung cancer. Here, a comprehensive review for the prediction of lung cancer by previous researcher using image processing techniques was presented. The summary for the prediction of lung cancer by previous researcher using image processing techniques was also presented.

\section{REFERENCES}

1. National Cancer Institute, "The cancer imaging archive, "https:1lwiki.cancerimagingarchive.net/display/Public/LIDC-lDRI, September 2011.

2. Sharma, D., \& Jindal, G. (2011). Identifying lung cancer using image processing techniques. In International Conference on Computational Techniques and Artificial Intelligence (ICCTAI'2011) (Vol. 17, pp. 872-880).

3. Tariq, A., Akram, M. U., \& Javed, M. Y. (2013, April). Lung nodule detection in CT images using neuro fuzzy classifier. In Computational Intelligence in Medical Imaging (CIMI), 2013 IEEE Fourth International Workshop on (pp. 49-53). IEEE.

4. Chaudhary, A., \& Singh, S. S. (2012, September). Lung cancer detection on CT images by using image processing. In Computing Sciences (ICCS), 2012 International Conference on (pp. 142146). IEEE.

5. Hashemi, A., Pilevar, A. H., \& Rafeh, R. (2013). Mass Detection in Lung CT Images Using Region Growing Segmentation and Decision Making Based on Fuzzy Inference System and Artificial Neural Network. International Journal of Image, Graphics and Signal Processing (IJIGSP), 5(6), 16.

6. Anand, S. V. (2010, October). Segmentation coupled textural feature classification for lung tumour prediction. In Communication Control and Computing Technologies (ICCCCT), 2010 IEEE International Conference on(pp. 518-524). IEEE.

7. Lee, S. L. A., Kouzani, A. Z., \& Hu, E. J. (2008, November). A random forest for lung nodule identification. In TENCON 2008-2008 IEEE Region 10 Conference (pp. 1-5). IEEE.

8. Zhang, F., Song, Y., Cai, W., Zhou, Y., Shan, S., \& Feng, D. (2013, November). Context curves for classification of lung nodule images. In Digital Image Computing: Techniques and Applications (DICTA), 2013 International Conference on (pp. 1-7). IEEE.

9. Sivakumar, S., \& Chandrasekar, C. (2013). Lung nodule detection using fuzzy clustering and support vector machines. International Journal of Engineering and Technology, 5(1), 179-185.

10. Jaffar, M. A., Hussain, A., Nazir, M., Mirza, A. M., \& Chaudhry, A. (2008, December). GA and morphology based automated segmentation of lungs from $\mathrm{Ct}$ scan images. In Computational Intelligence for Modelling Control \& Automation, 2008 International Conference on (pp. 265270). IEEE.

11. Madero Orozco, H., Vergara Villegas, O. O., De Jesus Ochoa Dominguez, H., \& Cruz Sanchez, V. G. (2013, November). Lung Nodule Classification in CT Thorax Images Using Support Vector 
Machines. In Artificial Intelligence (MICAI), 2013 12th Mexican International Conference on (pp. 277-283). IEEE.

12. PATIL, D. S., \& Kuchanur, M. B. (2012). Lung cancer classification using image processing. International Journal of Engineering and Innovative Technology (IJEIT) Volume, 2.

13. Naresh, P., \& Shettar, R. (2014). Image Processing and Classification Techniques for Early Detection of Lung Cancer for Preventive Health Care: A Survey. Int. J. of Recent Trends in Engineering \& Technology, 11.

14. Gajdhane, M. V. A., \& Deshpande, L. M. Detection of Lung Cancer Stages on CT scan Images by Using Various Image Processing Techniques.

15. Gomathi, M., \& Thangaraj, P. (2010). A computer aided diagnosis system for lung cancer detection using support vector machine. American Journal of Applied Sciences, 7(12), 1532.

16. Ilya Levner, Hong Zhangm(2007) ,"Classification driven Watershed segmentation ”, IEEE TRANSACTIONS ON IMAGE PROCESSING VOL. 16, NO. 5.

17. Sunil Kumar(2014), "Lung Segmentation using Region Growing Algorithm", International Journal of Advanced Research in Computer Science and Software Engineering Volume 4.

18. Fatma Taher, Naoufel Werghi and Hussain Al-Ahmad (2012), "Bayesian Classification and Artificial Neural Network Methods for Lung Cancer Early Diagnosis", IEEE.

19. JIA Tong, ZHAO Da-Zhe, YANG Jin-Zhu,WANG Xu (2007), "Automated Detection of Pulmonary Nodules in HRCT Images", IEEE.

20. Kesav Kancherla, Srinivas Mukkamala (2007), "Early Lung Cancer Detection using Nucleus Segmentation based Features", IEEE Symposium on Computational Intelligence in Bioinformatics and Computational Biology (CIBCB).

21. Amjed S Eslam b Jaber (2014)," Automated detection of lung cancer using statistical and morphological image processing techniques”.Journal of Biomedical Graphics and Computing, Vol. 4.

22. Ghayoumi H and Siamak J (2013)," Recognition and Classification of the Cancer Cells by using Image Processing and LabVIEW". International Journal of Computer Theory and Engineering, Vol. 5.

23. Ada (2013)," Feature Extraction and Principal Component Analysis for Lung Cancer Detection in CT scan Images". International journal of Advanced Research in Computer Science and Software Engineering, Vol. 3.

24. Sudha V (2012)," Lung Nodule Detection in CT Images using Thresholding and Morphological operations". International journal of Emerging Science and Engineering (IJESE), Vol. 1.

25. Lo, S.-C.B., Lou, S.-L.A., Lin, J.-S., Freedman, M.T., Chien, M.V. and Mun, S.K. (1995), "Artificial convolution neural network techniques and applications for lung nodule detection", IEEE T. Med. Imaging, Vol. 14 No. 4, pp. 711-8.

26. Yoshida, H., Xu, X.-W., Kobayashi, T., Giger, M.L. and Doi, K. (1995), "Computer-aided diagnosis scheme for detecting pulmonary nodules using wavelet transform”, Proc. of SPIE, Vol. 2434, p. 621.

27. Giger, M.L., Doi, K., MacMahon, H., Metz, C.E. and Yin, F.F. (1990), "Pulmonary nodules: computer-aided detection in digital chest images", Radiographics, Vol. 10 No. 1, p. 41.

28. De Boo, D.W., Prokop, M., Uffmann, M., van Ginneken, B. and Schaefer-Prokop, C.M. (2009), "Computer-aided detection (CAD) of lung nodules and small tumours on chest radiographs", Eur. J. Radiol., Vol. 72 No. 2, pp. 218-25.

29. de Hoop, B., De Boo, D.W., Gietema, H.A., van Hoorn, F., Mearadji, B., Schijf, L., van Ginneken, B., Prokop, M. and Schaefer-Prokop, C. (2010), "Computer-aided detection of lung cancer on chest radiographs: effect on observer performance", Radiology, Vol. 257 No. 2, p. 532.

30. Kobayashi, T., Xu, X.W., MacMahon, H., Metz, C.W. and Doi, K. (1996), "Effect of a computeraided diagnosis scheme on radiologists' performance in detection of lung nodules on radiographs", Radiology, Vol. 199, pp. 843-8.

31. MacMahon, H., Engelmann, R., Behlen, F.M., Hoffmann, K.R., Ishida, T., Roe, C., Metz, C.E. and Doi, K. (1999), "Computer-aided diagnosis of pulmonary nodules: results of a large-scale observer test”, Radiology, Vol. 213, pp. 723-6.

32. Muhm, J.R., Miller, W.E., Fontana, R.S., Sanderson, D.R. and Uhlenhopp, M.A. (1983), "Lung cancer detected during a screening program using four-month chest radiographs", Radiology, Vol. 148 No. 3, p. 609. 
33. Shah, P.K., Austin, J.H.M., White, C.S., Patel, P., Haramati, L.B., Pearson, G.D.N., Shiau, M.C. and Berkmen, Y.M. (2003), "Missed non-small cell lung cancer: radiographic findings of potentially resectable lesions evident only in retrospect1", Radiology, Vol. 226 No. 1.

34. Quekel, L.G., Kessels, A.G., Goei, R. and van Engelshoven, J.M. (1999), "Miss rate of lung cancer on the chest radiograph in clinical practice", Chest, Vol. 115 No. 3, p. 720.

35. Doi, K. (2007), "Computer-aided diagnosis in medical imaging: historical review, current status and future potential", Comput. Med. Imag. Grap., Vol. 31 Nos 4/5, pp. 198-211.

36. Xu, X.W., Doi, K., Kobayashi, T., MacMahon, H. and Giger, M.L. (1997), "Development of an improved CAD scheme for automated detection of lung nodules in digital chest images", Med. Phys., Vol. 24, p. 1395.

37. Penedo, M.G., Carreira, M.J., Mosquera, A. and Cabello, D. (1998), “Computer-aided diagnosis: a neural-network-based approach to lung nodule detection”, IEEE T. Med. Imaging, Vol. 17 No. 6, pp. 872-80.

38. Mao, F., Qian, W., Gaviria, J. and Clarke, L.P. (1998), "Fragmentary window filtering for multiscale lung nodule detection: preliminary study", Acad. Radiol., Vol. 5 No. 4, pp. 306-11.

39. Schilham, A.M.R., Van Ginneken, B. and Loog, M. (2006), "A computer-aided diagnosis system for detection of lung nodules in chest radiographs with an evaluation on a public database", Med. Image Anal., Vol. 10 No. 2, pp. 247-58.

40. Pereira, C.S., Fernandes, H., Mendonicc,a, A.M. and Campilho, A. (2007a), "Detection of lung nodule candidates in chest radiographs", Pattern Recognition and Image Analysis, pp. 170-7.

41. Le, K. (2011), "Chest X-ray analysis for computer-aided diagnostic", Advanced Computing CCSIT 2011, pp. 300-9.

42. Sundararajan, R., Xu, H., Annangi, P., Tao, X., Sun, X.W. and Mao, L. (2010), "Multiresolution support vector machine based algorithm for pneumoconiosis detection from chest radiographs", Biomedical Imaging: From Nano to Macro, 2010 IEEE International Symposium on, pp. 1317-20.

43. Gergely Orbán Gábor Horváth, (2012),"Algorithm fusion to improve detection of lung cancer on chest radiographs", International Journal of Intelligent Computing and Cybernetics, Vol. 5 Iss 1 pp. $111-144$.

44. Weng, Q., Sun, Y., Peng, X., Wang, S., Gu, L., Qiang, L. and Xu, J. (2009), "Computer-aided diagnosis: a support-vector-machine-based approach of automatic pulmonary nodule detection in chest radiographs", Proc. of the 2009 International Symposium on Bioelectronics and Bioinformatics, p. 60.

45. Snoeren, P.R., Litjens, G.J.S., Van Ginneken, B. and Karssemeijer, N. (2010), "Training a computer aided detection system with simulated lung nodules in chest radiographs", Proc. of The Third International Workshop on Pulmonary Image Analysis, p. 139.

46. Tuba kiyan, Tulay Yildirim(2004) "Breast cancer diagnosis using statistical neural networks", Journal of Electrical and Electronic Engineering.

47. Afzan Adam1 Khairuddin Omar2 "Computerized Breast Cancer Diagnosis with Genetic Algorithms AndNeuraNetworkfitt.mmu.edu.my/caiic/papers/afzaniCAIET

48. B.M.Gayathri, C.P.Sumathi (2013), " Breast Cancer Diagnosis Using Machine Learning Algorithm -A Survive". International Journal of Distributed and Parallel Systems (IJDPS) Vol.4, No. 3.

49. F.Paulin, A.Santhakumaran (2011)“Classification of Breast cancer by comparing Back propagation training algorithms", International Journal on Computer Science and Engineering (IJCSE).

50. Dr. K. Usha Rani ()" Parallel Approach for Diagnosis of Breast Cancer using Neural Network Technique" International Journal of Computer Applications, Volume 10- No.3.

51. David B.Fogel, Eugene C, Wasson, Edward M.Boughton (1995)“Evolving neural networks for detecting breast cancer". Elsevier Science Ireland Ltd.

52. L Rong (2010) ,'Diagnosis of Breast Tumor Using SVM-KNN Classifier” Intelligent Systems (GCIS).

53. Fatima Eddaoudi , Fakhita Regragui , Abdelhak Mahmoudi , Najib Lamouri (2011) "Masses Detection Using SVM Classifier Based on Textures Analysis"- Applied Mathematical Sciences, Vol. 5.

54. Aparna Kanakatte, Nallasamy Mani, Bala Srinivasan, Jayavardhana Gubbi (2008), "Pulmonary Tumor Volume Detection from Positron Emission Tomography Images", International Conference on Biomedical Engineering and Informatics. 
Advanced Computational Intelligence: An International Journal (ACII), Vol.3, No.1, January 2016

55. S. Aruna, Dr .S.P. Rajagopalan (2011), “ A Novel SVM based CSSFFS Feature Selection Algorithm for Detecting Breast Cancer” International Journal of Computer Applications (0975 8887) Volume 31 No.8.

56. Fatma Taher, Naoufel Werghi and Hussain Al-Ahmad (2012), "Bayesian Classification and Artificial Neural Network Methods for Lung Cancer Early Diagnosis”, IEEE.

57. Kesav Kancherla, Srinivas Mukkamala (2013), "Early Lung Cancer Detection using Nucleus Segmentation based Features", IEEE Symposium on Computational Intelligence in Bioinformatics and Computational Biology (CIBCB).

58. Negar Memarian, Javad Alirezaie, Paul Babyn (2006), "Computerized Detection of Lung Nodules with an Enhanced False Positive Reduction Scheme”, ICIP.

\section{Author}

Dr. Arvind Kumar Tiwari received the BE degree in Computer Science and Engineering from CCS university, Meerut., INDIA in 2003, and M. Tech. in Computer Science and Engineering from Uttar Pradesh Technical University, Lucknow and Ph.D. in Computer Science and Engineering from IIT (BHU), Varanasi, INDIA. He has worked as and Professor and Vice Principal in GGS College of Modern Technology, Kharar, Punjab, INDIA. His research interests include computational biology and pattern recognition.

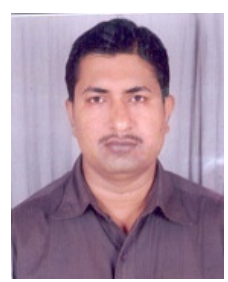

